\title{
Evaluation of Sperm DNA Fragmentation amongst Infertile Black Africans. A Nigerian Study
}

\author{
Abayomi B. Ajayi ${ }^{1}$, Bamgboye M. Afolabi ${ }^{2 *}$, Victor D. Ajayi ${ }^{1}$, Ifeoluwa 0. Oyetunji ${ }^{1}$, \\ Adedamilola Atiba', Seun Saanu' ${ }^{1}$, Ayodeji T. Adeoye ${ }^{1}$, Temilade E. Adeshida ${ }^{1}$, \\ Joy Ehichioya1, Ibukun I. Ayelehin'1 \\ ${ }^{1}$ Nordica Fertility Center, Lagos, Nigeria \\ ${ }^{2}$ Health, Environment and Development Foundation, Lagos, Nigeria \\ Email: `bmafolabi@gmail.com, ^heendef05@gmail.com
}

How to cite this paper: Ajayi, A.B., Afolabi, B.M., Ajayi, V.D., Oyetunji, I.O., Atiba, A., Saanu, S., Adeoye, A.T., Adeshida, T.E., Ehichioya, J. and Ayelehin, I.I. (2018) Evaluation of Sperm DNA Fragmentation amongst Infertile Black Africans. A Nigerian Study. Open Journal of Urology, 8, 297-316.

https://doi.org/10.4236/oju.2018.811034

Received: September 20, 2018

Accepted: November 11, 2018

Published: November 15, 2018

Copyright $(9) 2018$ by authors and Scientific Research Publishing Inc. This work is licensed under the Creative Commons Attribution International License (CC BY 4.0).

http://creativecommons.org/licenses/by/4.0/

\begin{abstract}
Background: Male infertility is approaching an epidemic proportion. Almost $50 \%$ of all cases of infertility may be associated with a male factor. The diagnostic usefulness of sperm DNA integrity is now accessible as an additional tool to Seminal Fluid Analysis. Objective: To assess sperm DNA fragmentation index (SDFI) in male infertility and its relationship with obesity, alcohol consumption and cigarette smoking among infertile Nigerians. Patients and Methods. Patients who presented for infertility at three health facilities of Nordica Fertility Center in Lagos, Asaba and Abuja cities in Nigeria. STATA 13 was used for student's t-test to compare the means of continuous variables among smokers and non-smokers and among alcohol consumers and non-consumers. Linear regression analysis was employed to assess the correlation between SDFI as dependent variable and some independent variables. Results. There was no significant difference in the SDFI of men aged $<40$ years compared to older men. There was also no significant difference in the proportion of men with SDFI of $<25 \%$ and of $\geq 25 \%$ regardless of their age group. The mean SDFI of men with normal BMI (30.8\%) was significantly lower $(\mathrm{t}=-1.80, \mathrm{P}$-value $=0.04)$ than that of obese men $(30.2 \%)$. Obese men were 2.12 times as likely to have SDFI $\geq 25 \%$ compared to normal weight men $\left(\chi^{2}-2.16, \mathrm{P}\right.$-value $\left.=0.14, \mathrm{OR}=2.12,95 \% \mathrm{CI}: 0.77,5.80\right)$. Mean SDFI of men who consume alcohol (37.1\%) was significantly higher $(\mathrm{t}=-1.97$, P-value $=$ 0.03) than that of those who did not consume alcohol. Although Pearson's correlation matrix $(r)$ indicated that sperm DNA fragmentation index was positively correlated with history of infertility $(r=0.01)$, groin surgery $(r=$ $0.04)$, mumps $(r=0.04)$ and sexually transmitted illness $(r=0.04)$, however the degree of correlation was not significant $(\mathrm{P}$-value $\geq 0.5)$ in each case.
\end{abstract}


Conclusion: This is the first report in Black Africa that describes a correlation between sperm DNA integrity, as measured by the halo test and age, BMI and alcohol consumption. Men with normal BMI were more likely to have excellent to good SDFI and hence good fertility potential. Data from this study indicate that the infertile men had significantly higher sperm DNA fragmentation. Obese men and those engaged in alcohol consumption also had higher sperm DNA fragmentation indices.

\section{Keywords}

Sperm Fragmentation Index, Obesity, Alcohol Consumption, Male Infertility, Assisted Reproduction Technology, Black Africans

\section{Introduction}

Male infertility is gradually becoming an epidemic, a phenomenon that is rising at an alarming rate. Inability to father a child can be a major stress-producing factor and a frustrating dilemma for either the man or the woman or as a matter of fact both the man and a woman in a conjugal relationship. In some cases, the relationship is pre-matrimonial where a man wants to be sure that the woman can bear a child for him. Regrettably, the blame of infertility is mostly on the female partner not being able to get pregnant. Globally, infertility has been observed to affect about $15 \%-20 \%$, an average of 1 in every 6 couples of child-producing age group. In approximately $40 \%$ of these cases, a male factor is involved, and this proportion reaches up to $60 \%$ where men are directly or partially responsible for the infertility [1]. The World Health Organization (WHO) defines male infertility as the presence of an alteration in concentration, motility and/or morphology in at least one sample of two sperm analyses [2]. Male infertility resulting from congenital or acquired urogenital abnormalities, malignancies, increased scrotal temperature (e.g. as a consequence of varicocele), endocrine disturbances, genetic abnormalities, and immunological factors [3] contribute to a definitive health seeking behavior among men, to raise a family. Although most men in the developed countries may be made aware, through screening or regular check-ups, of any of these causes of male infertility, men in sub-Saharan Africa are mostly unaware of risk factors for male infertility. Men often experience "waves of denial and shame, feeling like a failure" according to a report from a South African couple [4]. About 30\% of men in USA do not know why they are infertile [5] and probably many more so in Africa. Sperm DNA fragmentation test assesses the quantity of damaged DNA in a sample of seminal fluid with sperm cells. Karsian [5] emphasizes that all men have some amount of damage to their sperm DNA which implies that the higher the percentage of damage to DNA, the higher the chance of male infertility and the lesser the chance of achieving pregnancy. Thus, current diagnostic tools in male fertility, such as the conventional Seminal Fluid Analysis (SFA) and others, are 
insufficient [6] and unable to determine the quantity of damaged sperm DNA in an infertile man, being mainly based on the evaluation of sperm parameters such as concentration, motility and morphology [2]. The role of sperm DNA fragmentation (SDF) in male factor infertility has been emerging as a valuable tool for male infertility evaluation [7]. According to Bradley et al., SDF is used in assisted reproductive technology (ART) programs as an indicator for sperm quality, although there is still a lack of consensus as to its clinical utility [8]. A main contributing influence of sperm DNA mutilation is oxidative stress due to excessive production of reactive oxygen species [9] [10] [11] [12]. Other factors include defects in sperm chromatin packaging and DNA repair mechanisms as well as abnormalities in the regulation of programmed cell death (abortive apoptosis) which is vital for regulating sperm production [10] [11] [12]. Various studies have indicated that high sperm DNA fragmentation could also be linked with a diversity of exogenous factors such as infection, leucocytospermia, high fever, elevated testicular temperature (such as professional drivers), varicocele, advanced age, obesity, poor diet, drug use, cigarette smoking and exposure to high level of environmental and occupational pollutants [9] [11] [13] and to abstinence (infrequent ejaculation), trauma to the testicles and testicular cancer [14]. In recent years, there has been an upsurge in obesity pandemic in developed and developing countries as obesity has been associated with decreased fertility and could be considered as an etiological factor in male infertility [15]. Obesity, alcohol consumption, and tobacco have also been associated with DNA damage from increased oxidative stress [16]. Wdowiak et al. [17] reported that the burden of risky alcohol consumption result in an intensification of sperm DNA fragmentation [17] while Anifandis et al., [18] suggested that cigarette smoking and alcohol consumption separately and combined, have deleterious effect on sperm parameters and SDF. Sperm cells exist on microscopic level, yet they are built to carry enormous quantities of genetic information to the egg. There is a scarcity of Sperm DNA fragmentation studies in Africa. In the first 12 years of the $21^{\text {st }}$ Century, there were 2390 publications of Sperm DNA fragmentation [19], none from Africa even though in the past 10 - 15 years, a plethora of studies have confirmed that sperm DNA damage testing has strong associations with every early fertility check point [20]. These include impaired fertilization, slow early embryo development, reduced implantation, miscarriage and, in animal studies, birth defects in the offspring. Childhood cancers have also been associated with oxidative damage to sperm DNA because of paternal smoking [21]. The population of obese men is increasing in Africa as does the population of men who consume alcohol. The consequence of obesity and alcohol consumption has not been properly elucidated, at least from reproductive perspective. This study aims to explore Sperm DNA fragmentation among obese and alcohol-consuming Nigerian men with the objective of assess SDF among men with normal, overweight and obese BMI and also among those with different social habits. 


\section{Materials and Methods}

Sperm Chromatin Dispersion (SCD) testis based on the principle that when sperm immersed in an agarose matrix on a slide, treated with an acid solution to denaturate DNA, and then lysed with a commercial buffer solution to remove membranes and proteins, the result is the formation of nucleoids with a central core and a peripheral halo of dispersed DNA loops. The dispersion halos that are observed in sperm nuclei with non-fragmented DNA after the removal of nuclear proteins are either minimally present or not produced at all in sperm nuclei with fragmented DNA. The agarose matrix allows working with unfixed sperm on a slide in a suspension-like environment. Nucleoids are visualized either with fluorescent microscopy after staining with a DNA specific fluorochrome (DAPI), or with bright-field microscopy with Wright's staining solution. In this study, we performed the SCD test as proposed and improved upon by Fernandez et al., 2003 [22] using the Halosperm ${ }^{\oplus}$ kit (INDAS Laboratories, Madrid, Spain). In brief, an aliquot of a semen sample was diluted to $10 \mathrm{million} / \mathrm{mL}$ in phosphate-buffered saline (PBS). Gelled aliquots of low-melting point agarose in Eppendorf tubes were provided in the kit, each one to process a semen sample. Eppendorf tubes were placed in a water bath at $90^{\circ} \mathrm{C}-100^{\circ} \mathrm{C}$ for $5 \mathrm{~min}$ to fuse the agarose, and then in a water bath at $37^{\circ} \mathrm{C}$. After $5 \mathrm{~min}$ of incubation for temperature equilibration at $37^{\circ} \mathrm{C}, 60 \mathrm{~mL}$ of the diluted semen sample was added to the Eppendorf tube and mixed with the fused agarose. Of the semen-agarose mix, 20 $\mu \mathrm{L}$ was pipetted onto slides pre-coated with agarose, provided in the kit, and covered with a $22 \times 22 \mathrm{~mm}$ coverslip. The slides were placed on a cold plate in the refrigerator $\left(4^{\circ} \mathrm{C}\right)$ for $5 \mathrm{~min}$ to allow the agarose to produce a microgel with the sperm cells embedded within. The coverslips were gently removed, and the slides immediately immersed horizontally in an acid solution, previously prepared by mixing $80 \mu \mathrm{L}$ of $\mathrm{HCl}$ from an Eppendorf tube in the kit with $10 \mathrm{~mL}$ of distilled water, and incubated for $7 \mathrm{~min}$. The slides were horizontally immersed in $10 \mathrm{~mL}$ of the lysing solution for $25 \mathrm{~min}$. After washing for $5 \mathrm{~min}$ in a tray with abundant distilled water, the slides were dehydrated in increasing concentrations of ethanol (70\%, 90\%, and 100\%) for 2 min each and then air-dried. For bright field microscopy, slides were horizontally covered with a mix of Wright's staining solution (Merck, Darmstadt, Germany) and PBS (Merck) (1:1) for 5 - 10 min with continuous airflow. Slides were briefly washed in tap water and allowed to dry.

Four dispersion patterns were defined: 1) Sperm with large halo: the halo has a 2 times larger width than that of the sperm core, with a darker spot (sperm head) in the middle 2) Sperm with moderate halo: having a halo size between large and small halos 3) Sperm with small halo: a very small, clear film, that has a halo appearance, surrounds the sperm head 4) Sperm with no halo. Sperm DNA fragmentation percentage (or SCD percentage) was calculated as the proportion of sperm with big, small and no halos, to the total sperm count per slide. We assessed two slides for every patient, and a total of 1000 sperms were counted per 
slide. The halos correspond to relaxed DNA loops attached to the residual nuclear structure [22]. The spermatozoa without DNA fragmentation show halos of dispersed DNA which can be large (big halo, bh) or medium (medium halo, $\mathrm{mh}$ ), whereas those sperm nuclei with fragmented DNA produce either small halos (small halo, sh) or no halos at all. Initial laboratory results were reported, according to natural and IUI conceptions, showing 4 statistical categories of fertility potential: $<15 \%$ SDFI $=$ excellent to good sperm DNA integrity; $\geq 15$ to $<25 \%$ SDFI = good to fair sperm DNA integrity; $\geq 25 \%$ to $<50 \%$ SDFI $=$ fair to poor sperm DNA integrity; and $\geq 50 \%$ SDFI = very poor sperm DNA integrity [23]. Statistically significant threshold for subfertility had been established at SDFI $>25 \%$. Age (years) was categorized into $<40$ and $\geq 40$ years and Body Mass Index $\left(\mathrm{kg} / \mathrm{m}^{2}\right)$ into normal (BMI of 18.5 - 24.5), overweight (BMI of 25 - 29.9) and obese (BMI of $\geq 30$ ). After resting for at least 10 minutes, electronic sphygmomanometer was, in the left upper brachium, used to measure systolic and diastolic blood pressure of each patient twice and the mean was recorded. An open-ended questionnaire was the instrument used to collect subjects' socio-demographic data as well as medical and surgical history such as hypertension, diabetes, torsion of the testes, mumps, groin surgery, varicocele, sexually transmitted illness and undescended testis.

\section{Statistical Analysis}

Sperm DNA fragmentation index (SDFI) in this study was categorized as $<25 \%$ or good SDF and $\geq 25 \%$ as bad SDFI. Statistical analysis on the data collected in this study was performed using STATA 13 for Windows (Stata Corps, College Station, Texas 77845, USA). All variables were initially tested to determine variance homogeneity and data normality, and heteroscedastic data were transformed. Groups were compared using one-way ANOVA. Analyses carried out included frequency and percentage of proportions, appropriate bivariate (crosstabulation) and multivariate regression analysis. Statistical variances between means were decided by Student's t-test when comparing 2 groups and by Kruskal-Wallis where comparing more than 2 groups. Outcomes were given as mean ( \pm standard deviation [sd]). The significance of differences between two or more than two proportions was determined using Chi-square $\left(\chi^{2}\right)$ test. Odds ratio was determined at $95 \%$ Confidence Interval. Data were presented as figures, tables and graphs. Association between sperm DNA fragmentation index and continuous variables such as age, BMI, systolic and diastolic blood pressures was assessed using Linear Regression Analysis. Level of significance was set at $\mathrm{P}<$ 0.05 .

\section{Ethics Approval}

This study was approved by the State Ethics Committee on Health Research.

\section{Results}

A total of 111 apparently health males were involved in the study which took 
place between January 4 and December 3 of 2017 among patients who presented at Nordica Fertility Center (NFC). The means ( \pm ) of age (years) and body mass index $\left(\mathrm{kg} / \mathrm{m}^{2}\right)$ were $40.3(8.88)$ and $27.4(4.7)$ respectively. Fifty-four $(48.6 \%)$ of the subjects were aged $<40$ years and $57(51.4 \%)$ were 40 years or older. In all, 39 (35.1\%), 42 (37.9\%) and 30 (27.0\%) of the study subjects were normal in BMI, overweight or obese. The means $( \pm)$ of systolic and diastolic Blood Pressure $(\mathrm{mm} \cdot \mathrm{Hg})$ were $131.0(17.2)$ and $83.6(11.5)$ respectively. A total of $34(30.6 \%)$ and $40(36.0 \%)$ had systolic and diastolic hypertension of $\geq 140 / \geq 90 \mathrm{~mm} \cdot \mathrm{Hg}$ while $77(69.4 \%)$ and $71(64.0 \%)$ did not have systolic or diastolic hypertension respectively. Only 4 (3.6\%) had a history of diabetes but 92 (82.9\%) had a history of male infertility. The study subjects were from different geo-political zones of the country: $42(37.8 \%)$ from the Southwest, 40 (36.0\%) from the Southeast and only 5 (4.5\%) were from the Northwest zone of the country (Table 1 ).

Figure 1 a graphical representation of age ( $x$-axis) against SDFI \% ( $y$-axis) of study subject, shows sperm DNA fragmentation as early as 22 years of age (subject

Table 1. Socio-demographic and medical characteristics of study subjects.

\begin{tabular}{|c|c|c|c|c|c|c|}
\hline Variable & Unit & Freq. & Percent & Mean & $\pm \mathrm{sd}$ & Range \\
\hline \multirow{3}{*}{ Age (years) } & All & 111 & 100.0 & 40.3 & 8.8 & $22-65$ \\
\hline & $<40$ & 54 & 48.6 & 33.2 & 4.8 & $22-39$ \\
\hline & $\geq 40$ & 57 & 51.4 & 47.1 & 6.0 & $40-65$ \\
\hline \multirow{4}{*}{ BMI $\left(\mathrm{kg} / \mathrm{m}^{2}\right)$} & All & 111 & 100.0 & 27.4 & 4.7 & $18.7-45.7$ \\
\hline & $18.5-24.9$ & 39 & 35.1 & 22.8 & 1.5 & $18.7-24.9$ \\
\hline & $25.0-29.9$ & 42 & 37.9 & 27.4 & 1.3 & $25.0-29.6$ \\
\hline & $\geq 30$ & 30 & 27.0 & 33.4 & 3.4 & $30.1-45.7$ \\
\hline \multirow{3}{*}{$\begin{array}{c}\text { Systolic BP } \\
(\mathrm{mm} \cdot \mathrm{Hg})\end{array}$} & All & 111 & 100.0 & 131.0 & 17.2 & $90-178$ \\
\hline & $\geq 140$ & 34 & 30.6 & 150.2 & 11.6 & $140-178$ \\
\hline & $<140$ & 77 & 69.4 & 122.6 & 11.5 & $90-139$ \\
\hline \multirow{3}{*}{$\begin{array}{c}\text { Diastolic BP } \\
(\mathrm{mm} \cdot \mathrm{Hg})\end{array}$} & All & 110 & 100.0 & 83.6 & 11.5 & $63-123$ \\
\hline & $\geq 90$ & 40 & 36.0 & 95.6 & 8.1 & $90-123$ \\
\hline & $<90$ & 71 & 64.0 & 76.8 & 6.5 & $63-89$ \\
\hline \multirow{2}{*}{$\begin{array}{c}\text { History of } \\
\text { Diabetes }\end{array}$} & Yes & 4 & 3.6 & - & - & - \\
\hline & No & 107 & 96.4 & - & - & - \\
\hline \multirow{5}{*}{$\begin{array}{l}\text { History of } \\
\text { infertility }\end{array}$} & Yes & 92 & 82.9 & - & - & - \\
\hline & No & 19 & 17.1 & - & - & - \\
\hline & Southwest & 42 & 37.8 & - & - & - \\
\hline & Southeast & 40 & 36.0 & - & - & - \\
\hline & South-south & 22 & 19.8 & - & - & - \\
\hline \multirow[t]{4}{*}{ Zone of origin } & Northcentral & 5 & 4.5 & - & - & - \\
\hline & Northwest & 1 & 0.90 & - & - & - \\
\hline & Northeast & 0 & 0.0 & - & - & - \\
\hline & Others & 1 & 0.90 & - & - & - \\
\hline
\end{tabular}




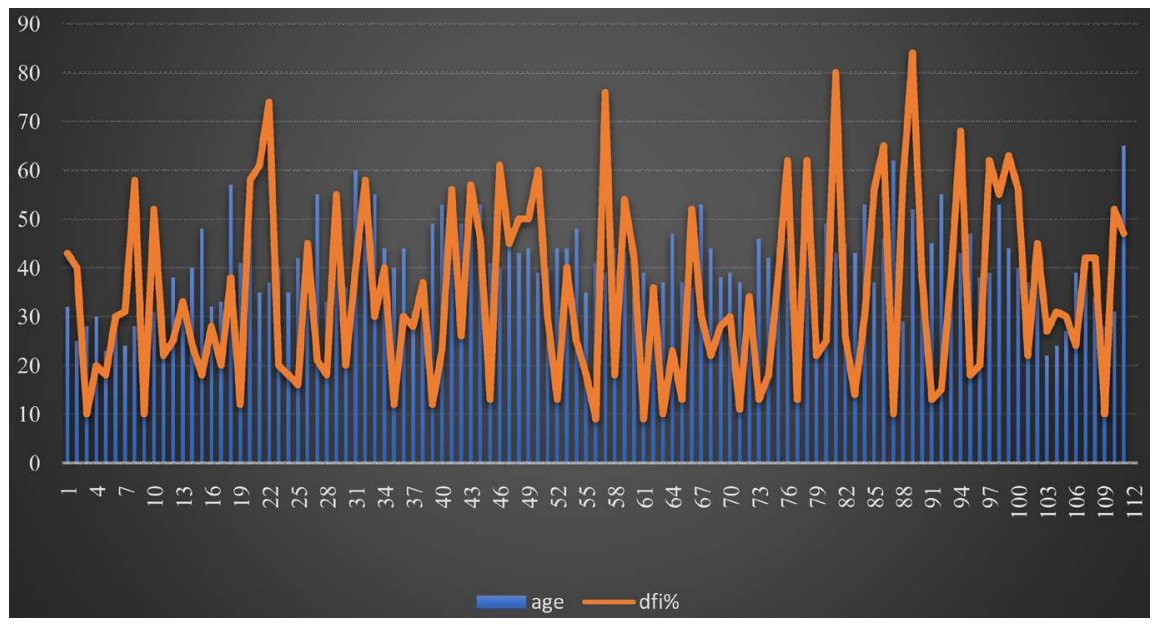

Figure 1. Combined bar and line graph of DNA fragmentation index (\%) relative to age of study subjects.

$104)$ with a SDFI of $27 \%$ and as late as about 65 years of age with a SDFI of $47 \%$ (subject 111). The highest sperm DNA fragmentation index (84\%) was at age 52 years (subject 89) and the lowest (9\%) was aged 41 (subject 56).

Likewise, Figure 2 shows the graphical illustration of BMI (x-axis) against SDFI\% (y-axis) of study subjects. The highest SDFI of $84 \%$ has a BMI of 30.3 $\mathrm{kg} / \mathrm{m}^{2}$ while two subjects had the lowest SDFI of $9 \%$, one with a BMI of 24.5 $\mathrm{kg} / \mathrm{m}^{2}$ and the other with a BMI of $28.5 \mathrm{~kg} / \mathrm{m}^{2}$. The highest BMI $\left(45.7 \mathrm{~kg} / \mathrm{m}^{2}\right)$ had a SDFI of $20 \%$ while the lowest BMI $\left(18.72 \mathrm{~kg} / \mathrm{m}^{2}\right)$ had a SDFI of $56 \%$.

The mean $( \pm$ ) DNA fragmentation index (SDFI\%) of all the study subjects was 34.5 (18.7). A total of 21 (38.9\%) men aged $<40$ years had a SDFI $<25 \%$ while 33 (61.1\%) men of the same age had a SDFI $\geq 25 \%$. On the other hand, $24(42.1 \%)$ men aged 40 years and older had SDFI $<25 \%$ while 33 (57.9\%) had SDFI $\geq 25 \%$. There was no significant difference in the mean SDFI\% of the two age groups of study subjects. When BMI was considered, the odds of overweight subjects having SDFI $<25 \%$ was 0.79 times $\left(\chi^{2}=0.27, \mathrm{P}\right.$-value $=0.61$, OR $=0.79,95 \% \mathrm{CI}$ : $0.33,1.91)$ and obese subjects had even a more remote odd of 0.47 to be included in SDFI of $<25 \%\left(\chi^{2}=2.16\right.$, P-value $=0.14, \mathrm{OR}=0.47,95 \%$ CI: $\left.0.17,1.29\right)$. On the contrary, the probability of overweight men having a SDFI $\geq 25 \%$ was 1.26 $\left(\chi^{2}=0.27, \mathrm{P}\right.$-value $\left.=0.61, \mathrm{OR}=1.26,95 \% \mathrm{CI}: 0.52,3.04\right)$ while obese men had a higher probability of $2.12\left(\chi^{2}=2.16, \mathrm{P}\right.$-value $=0.14$, OR $=2.12,95 \%$ CI: 0.77 , $5.80)$. There was a statistically significant difference $(t=-1.80, \mathrm{P}$-value $=0.04)$ in the mean SDFI\% of subjects with normal BMI $(30.8 \pm 16.1)$ compared to that of obese subjects ( $39.2 \pm 21.3$ ) (Table 2).

Table 3 and Figure 3 show the SDFI\% of the subjects according to their social habits. Of the 111 men in the study, 69 (61.3\%) consume alcohol, either regularly or occasionally, whose SDFI\% $(37.1 \pm 19.2)$ was significantly $(\mathrm{t}=1.97$, P-value $=$ $0.03)$ higher than that of non-consumers of alcohol (30.2 \pm 17.1$)$. Only $2(1.8 \%)$ men reported use of cannabis sativa (C. sativa). Surprisingly, the SDFI\% of these two were significantly lower than that of those who reported non-use of $C$. sativa. 


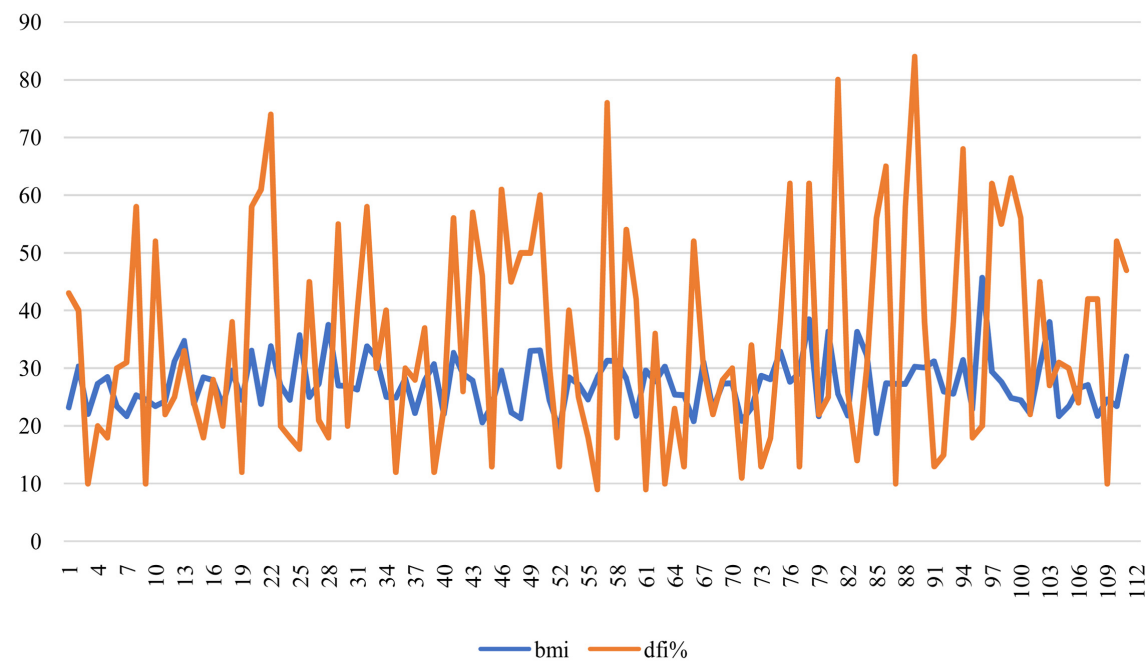

Figure 2. Line graph of DNA fragmentation index (\%) relative to BMI of study subjects.

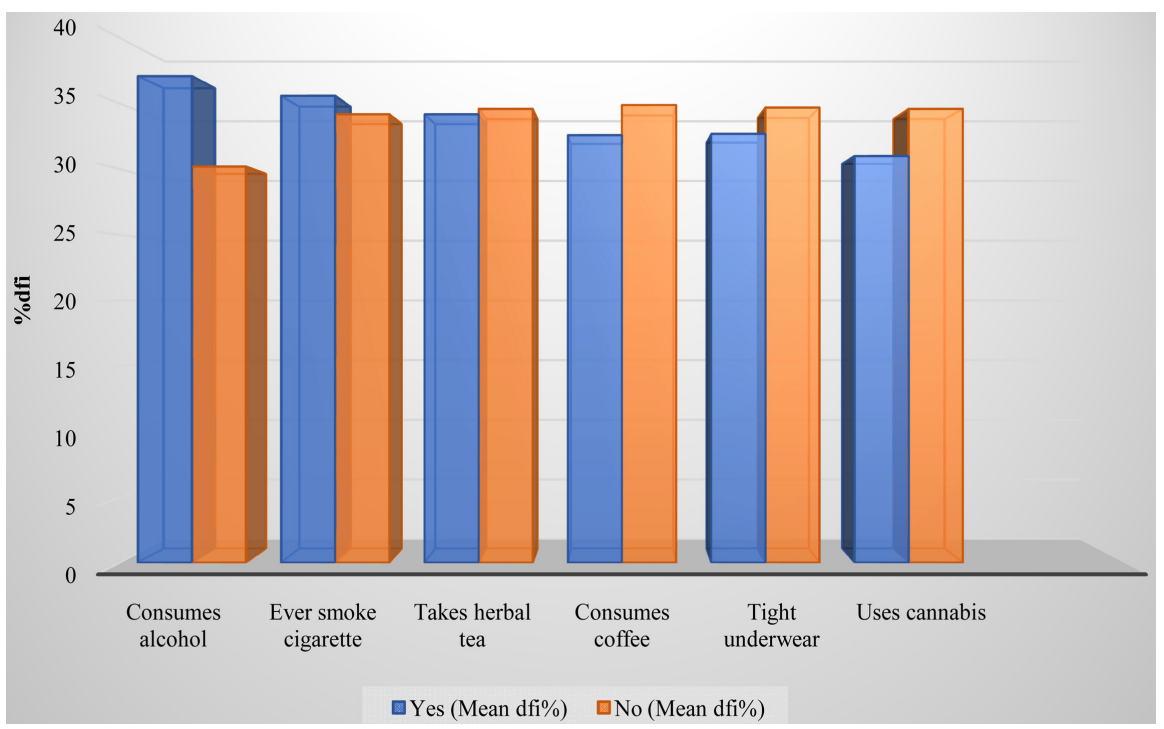

Figure 3. Mean DNA fragmentation index of subjects relative to their social habits.

However, the small number of respondent warrants a note of caution in interpreting this result. Those who take herbal teas ( $\mathrm{OR}=0.67,95 \%$ CI: $0.29,1.50)$, consume alcohol $(\mathrm{OR}=0.73,95 \% \mathrm{CI}: 0.34,1.59)$ or caffeine $(\mathrm{OR}=0.75,95 \% \mathrm{CI}$ : $0.27,2.06$ ) were unlikely to fall into the group of SDFI $<25 \%$ whereas those who did not take herbal teas (OR $=1.50,95 \%$ CI: $0.66,3.39)$, consume alcohol (OR = $1.37,95 \%$ CI: $0.63,2.98)$, or caffeine ( $\mathrm{OR}=1.33,95 \%$ CI: $0.49,3.65)$, were 1.50 , 1.37 , and 1.33 times as likely to fall within the group of SDFI $<25 \%$ i.e. good SDFI. There was no significant difference in the mean SDFI\% of those who smoke cigarette (35.6\%) and those who do not (34.2\%). Surprisingly, those who did not smoke cigarette were observed to be unlikely to fall in the SDFI $<25 \%$ group ( $\mathrm{OR}=0.86,95 \% \mathrm{CI}: 0.34,2.17)$, putting those who did smoke into higher odds (OR $=1.16$; $95 \%$ CI: $0.46,2.94)$ of being in SDFI $<25 \%$ group. It was also observed that those with systolic blood pressure $\geq 140 \mathrm{~mm} \mathrm{Hg}$ were unlikely to 
Table 2. Frequency distribution, means and linear regression analysis of Age and BMI of study subjects relative to SDFI\%.

(a)

\begin{tabular}{|c|c|c|c|c|c|c|c|c|c|}
\hline \multirow[b]{2}{*}{ Age (years) } & \multicolumn{3}{|c|}{ SDFI $<25 \%$} & \multicolumn{3}{|c|}{ SDFI $\geq 25 \%$} & \multicolumn{3}{|c|}{ SDFI\% } \\
\hline & All & $<40$ & $\geq 40$ & $<40$ & $\geq 40$ & All & $<40$ & $\geq 40$ & All \\
\hline Freq. & 45 & 21 & 24 & 33 & 33 & 66 & 54 & 57 & 111 \\
\hline$\%$ & 40.5 & 38.9 & 42.1 & 61.1 & 57.9 & 59.5 & 48.6 & 51.4 & 100.0 \\
\hline Mean & 16.9 & 16.9 & 17.0 & 43.6 & 49.4 & 46.5 & 33.2 & 35.7 & 34.5 \\
\hline $\pm s d$ & 5.1 & 5.3 & 5.0 & 13.6 & 15.1 & 14.6 & 17.2 & 20.0 & 18.7 \\
\hline$\chi^{2}$ & - & \multicolumn{4}{|c|}{0.12} & - & - & - & - \\
\hline P-value & - & \multicolumn{4}{|c|}{0.73} & - & - & - & - \\
\hline Odds Ratio & - & \multicolumn{4}{|c|}{0.88} & - & - & - & - \\
\hline $95 \%$ CI & - & \multicolumn{4}{|c|}{$0.41,1.87$} & - & - & - & - \\
\hline t-test & - & \multicolumn{2}{|c|}{-0.07} & \multicolumn{2}{|c|}{-1.64} & - & \multicolumn{2}{|c|}{-0.71} & - \\
\hline $\mathrm{P}$-value & - & \multicolumn{2}{|c|}{0.47} & \multicolumn{2}{|c|}{0.05} & - & \multicolumn{2}{|c|}{0.24} & - \\
\hline
\end{tabular}

(b)

\begin{tabular}{ccccccccccc}
\hline & \multicolumn{3}{c}{ SDFI $<25 \%$} & \multicolumn{3}{c}{ SDFI $\geq 25 \%$} & \multicolumn{3}{c}{ SDFI\% } \\
\cline { 2 - 10 } BMI $\left(\mathrm{kg} / \mathrm{m}^{2}\right)$ & $18.5-$ & $25.0-29.9$ & $\geq 30$ & $18.5-24.5$ & $25.0-$ & 29.9 & $\geq 30$ & $18.5-$ & $25.0-$ & 24.5 \\
& 24.5 & 17 & 10 & 21 & $25 !$ & 20 & 39 & 42 & 30 \\
\hline Freq. & 18 & 17 & 22.2 & 31.8 & 37.9 & 33.3 & 35.1 & 37.9 & 27.0 \\
$\%$ & 40.0 & 37.8 & 17.1 & 42.9 & 46.6 & 50.2 & 30.8 & 34.6 & 39.2 \\
Mean & 16.7 & 17.5 & 17.1 & \\
\pm sd & 5.2 & 5.2 & 5.1 & 11.7 & 14.2 & 17.1 & 16.1 & 18.6 & 21.3 \\
$\chi^{2}$ & 0.27 & 0.27 & 2.16 & 0.27 & 0.27 & 2.16 & - & - & - \\
P-value & 0.61 & 0.61 & 0.14 & 0.61 & 0.61 & 0.14 & - & - & - \\
Odds Ratio & & 0.79 & 0.47 & - & 1.26 & 2.12 & - & - & - \\
95\% CI & & $0.33,1.91$ & $0.17,1.29$ & - & $0.52,3.04$ & $0.77,5.80$ & - & - & - \\
t-test & - & -0.45 & -0.20 & - & -0.97 & -1.59 & - & -0.99 & -1.80 \\
P-value & - & 0.33 & 0.42 & - & 0.17 & 0.06 & - & 0.16 & 0.04 \\
\hline
\end{tabular}

have SDFI $<25 \%$ (OR $=0.87,95 \%$ CI: $0.38,1.99)$ but 1.15 more likely to have bad SDFI $\geq 25 \%$. Likewise, those with diastolic blood pressure $\geq 90 \mathrm{~mm} \mathrm{Hg}$ were unlikely (OR $=0.69,95 \%$ CI: $0.31,1.55)$ to fall into the SDFI $<25 \%$ group but were 1.44 times more likely to have bad SDFI $\geq 25 \%$, thus suggesting high blood pressure is associated high sperm DNA fragmentation. However, there was no linear relationship between SDFI and systolic or diastolic blood pressure as shown by separate scatter plots in Figure 4(a) and Figure 4(b).

As illustrated in Table 4, the odds of those with history of varicocele to fall into the SDFI <25\% (good SDFI) group was slim at $0.28(\mathrm{OR}=0.28,95 \% \mathrm{CI}$ : $0.03,2.46$ ), whereas the odds to be in the SDFI $\geq 25 \%$ group (bad SDFI) was high at $3.61(\mathrm{OR}=3.61,95 \% \mathrm{CI}: 0.41,31.96)$. Similarly, the odds of subjects with 
Table 3. Means of DNA fragmentation index (\%) associated with systolic and diastolic blood pressure $(\mathrm{mm} \cdot \mathrm{Hg})$, social habits and person behavioral characteristics.

\begin{tabular}{|c|c|c|c|c|c|c|c|c|c|c|c|c|c|c|c|}
\hline \multirow{2}{*}{ Variable } & \multirow{2}{*}{ Item } & \multirow{2}{*}{ Freq. } & \multirow{2}{*}{$\%$} & \multicolumn{4}{|c|}{ SDFI\% } & \multicolumn{2}{|c|}{ SDFI $<25 \%$} & \multicolumn{2}{|c|}{ SDFI $\geq 25 \%$} & \multirow{2}{*}{$\chi^{2}$} & \multirow{2}{*}{$\mathrm{P}$-value } & \multirow{2}{*}{$\begin{array}{l}\text { Odds } \\
\text { Ratio }\end{array}$} & \multirow{2}{*}{$95 \%$ CI } \\
\hline & & & & Mean & $\pm s d$ & t-test & $\mathrm{P}$-value & Freq. & $\%$ & Freq. & $\%$ & & & & \\
\hline \multirow{2}{*}{ Herbal teas } & Yes & 38 & 32.4 & 34.2 & 17.6 & \multirow{2}{*}{-0.11} & \multirow{2}{*}{0.46} & 13 & 28.9 & 25 & 37.9 & \multirow{2}{*}{0.96} & \multirow{2}{*}{0.33} & 0.67 & $0.29,1.50$ \\
\hline & No & 73 & 67.6 & 34.6 & 19.3 & & & 32 & 71.1 & 41 & 62.1 & & & 1.50 & $0.66,3.39$ \\
\hline \multirow{2}{*}{ Alcohol } & Yes & 69 & 61.3 & 37.1 & 19.2 & \multirow{2}{*}{1.97} & \multirow{2}{*}{0.03} & 26 & 57.8 & 43 & 65.2 & \multirow{2}{*}{0.62} & \multirow{2}{*}{0.43} & 0.73 & $0.34,1.59$ \\
\hline & No & 42 & 38.7 & 30.2 & 17.1 & & & 19 & 42.2 & 23 & 34.8 & & & 1.37 & $0.63,2.98$ \\
\hline \multirow{2}{*}{ Cigarette } & Yes & 23 & 21.6 & 35.6 & 16.8 & \multirow{2}{*}{0.34} & \multirow{2}{*}{0.37} & 10 & 22.2 & 13 & 19.7 & \multirow{2}{*}{0.10} & \multirow{2}{*}{0.75} & 1.16 & $0.46,2.94$ \\
\hline & No & 88 & 78.4 & 34.2 & 19.2 & & & 35 & 77.8 & 53 & 80.3 & & & 0.86 & $0.34,2.17$ \\
\hline \multirow{2}{*}{ Caffeine } & Yes & 20 & 18.0 & 32.6 & 19.1 & \multirow{2}{*}{-0.49} & \multirow{2}{*}{0.31} & 7 & 15.6 & 13 & 19.7 & \multirow{2}{*}{0.31} & \multirow{2}{*}{0.58} & 0.75 & $0.27,2.06$ \\
\hline & No & 91 & 82.0 & 34.9 & 18.7 & & & 38 & 84.4 & 53 & 80.3 & & & 1.33 & $0.49,3.65$ \\
\hline \multirow{2}{*}{$\begin{array}{c}\text { Tight } \\
\text { underwear }\end{array}$} & Yes & 9 & 8.1 & 32.7 & 22.1 & 02 & 040 & 5 & 11.1 & 4 & 6.1 & $026 x$ & $0-5$ & 1.94 & $0.49,7.65$ \\
\hline & No & 102 & 91.9 & 34.7 & 18.5 & $-0 . \angle 0$ & 0.40 & 40 & 88.9 & 62 & 93.9 & 0.50 & 0.53 & 0.51 & $0.13,2.04$ \\
\hline Use of & Yes & 2 & 1.8 & 31.0 & 0.0 & & $0 \cap 0$ & 0 & 0.0 & 2 & 3.0 & $020 x$ & 0 & - & undefined \\
\hline cannabis & No & 109 & 98.2 & 34.6 & 18.8 & -1.50 & $0.0 J$ & 45 & 100.0 & 64 & 97.0 & 0.20 & $0.0 J$ & - & Undefined \\
\hline Systolic & Yes & 34 & 30.6 & 38.3 & 20.6 & 125 & م00 & 13 & 28.9 & 21 & 31.8 & 01 & 77 & 0.87 & $0.38,1.99$ \\
\hline $\mathrm{BP} \geq 140$ & No & 77 & 69.4 & 32.8 & 17.6 & 1.35 & 0.09 & 32 & 71.1 & 45 & 68.2 & 0.11 & 0.74 & 1.15 & $0.50,2.63$ \\
\hline Diastolic & Yes & 40 & 36.0 & 36.5 & 19.5 & 0 70 & 1 & 14 & 31.1 & 26 & 39.4 & م & (2) & 0.69 & $0.31,1.55$ \\
\hline $\mathrm{BP} \geq 90$ & No & 71 & 64.0 & 33.5 & 18.2 & 0.19 & 0.21 & 31 & 68.9 & 40 & 60.6 & 0.80 & 0.37 & 1.44 & $0.65,3.21$ \\
\hline
\end{tabular}

Table 4. Frequency distribution, mean SDFI\% and odds of falling into good or bad SDFI among subjects with history of some surgical and medical conditions.

\begin{tabular}{|c|c|c|c|c|c|c|c|c|c|c|c|c|c|c|c|c|}
\hline \multirow{2}{*}{$\begin{array}{l}\stackrel{0}{0} \\
\stackrel{\Xi}{0} \\
00\end{array}$} & \multirow{2}{*}{ 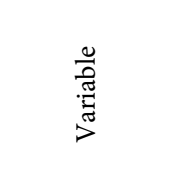 } & \multirow{2}{*}{ 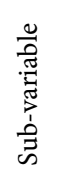 } & \multirow{2}{*}{ 离 } & \multirow{2}{*}{$a^{\circ}$} & \multirow{2}{*}{ 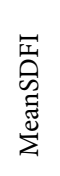 } & \multirow{2}{*}{$\begin{array}{l}\vec{c} \\
0 \\
+1\end{array}$} & \multirow{2}{*}{ 苞 } & \multirow{2}{*}{ 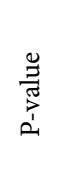 } & \multicolumn{2}{|c|}{ SDFI $<25 \%$} & \multicolumn{2}{|c|}{ SDFI $\geq 25 \%$} & \multirow{2}{*}{$\stackrel{N}{x}$} & \multirow{2}{*}{ 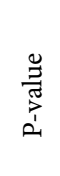 } & \multirow{2}{*}{ 뜽 } & \multirow{2}{*}{ 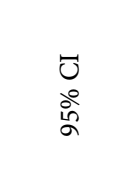 } \\
\hline & & & & & & & & & Freq. & $\%$ & Freq. & $\%$ & & & & \\
\hline \multirow{6}{*}{$\begin{array}{c}\text { Surgical } \\
\text { history }\end{array}$} & \multirow{2}{*}{$\begin{array}{l}\text { Testicular } \\
\text { torsion }\end{array}$} & Yes & 2 & 1.8 & 45.5 & 21.9 & \multirow[b]{2}{*}{0.72} & \multirow[b]{2}{*}{0.30} & 0 & 0.0 & 2 & 3.0 & \multirow[b]{2}{*}{020} & \multirow[b]{2}{*}{0.65} & \multirow{2}{*}{0.00} & \multirow{2}{*}{ undefined } \\
\hline & & No & 109 & 98.2 & 34.3 & 18.7 & & & 45 & 100.0 & 64 & 97.0 & & & & \\
\hline & \multirow{2}{*}{$\begin{array}{l}\text { Undescended } \\
\text { testis }\end{array}$} & Yes & 0 & 0.0 & - & - & \multirow{2}{*}{ - } & \multirow{2}{*}{ - } & 0 & 0.0 & 0 & 0.0 & - & - & - & - \\
\hline & & No & 111 & 100.0 & 34.5 & 18.7 & & & 45 & 100.0 & 66 & 100.0 & - & - & - & - \\
\hline & \multirow{2}{*}{ Varicocele } & Yes & 6 & 5.4 & 32.3 & 7.9 & \multirow{2}{*}{0.62} & \multirow{2}{*}{0.28} & 1 & 2.2 & 5 & 7.6 & \multirow{2}{*}{0.64} & \multirow{2}{*}{042} & 0.28 & $0.03,2.46$ \\
\hline & & No & 105 & 94.6 & 34.6 & 19.1 & & & 44 & 97.8 & 61 & 92.4 & & & 3.61 & $0.41,31.96$ \\
\hline \multirow{8}{*}{$\begin{array}{l}\text { Medical } \\
\text { history }\end{array}$} & \multirow{2}{*}{ Hypertension } & Yes & 35 & 31.5 & 36.8 & 19.8 & & & 14 & 31.1 & 21 & 31.8 & & & 0.97 & $0.43,2.19$ \\
\hline & & No & 76 & 68.5 & 33.4 & 10.2 & 0.00 & 0.17 & 31 & 68.9 & 45 & 68.2 & 0.01 & דינ. & 1.03 & $0.46,2.34$ \\
\hline & Diahetes & Yes & 4 & 3.6 & 36.8 & 7.9 & 055 & 030 & 1 & 2.2 & 3 & 4.5 & 002 & 090 & 0.48 & $0.05,4.74$ \\
\hline & & No & 107 & 96.4 & 34.4 & 19.0 & & & 44 & 97.8 & 63 & 95.5 & & & 2.10 & $0.21,20.81$ \\
\hline & Sexually & Yes & 21 & 18.6 & 36.4 & 18.6 & & & 8 & 17.8 & 13 & 19.7 & & & 0.88 & $0.33,2.34$ \\
\hline & Illness & No & 90 & 81.1 & 34.1 & 18.7 & 0.01 & 0.31 & 37 & 82.2 & 53 & 90.3 & 0.00 & 0.00 & 1.13 & $0.43,3.01$ \\
\hline & & Yes & 6 & 5.4 & 37.5 & 25.3 & & & 2 & & 4 & & & & 0.72 & $0.13,4.11$ \\
\hline & Mumps & No & 105 & 94.6 & 34.3 & 18.4 & 0.31 & 0.39 & 43 & & 62 & & 0.00 & 1.00 & 1.39 & $0.24,7.91$ \\
\hline
\end{tabular}




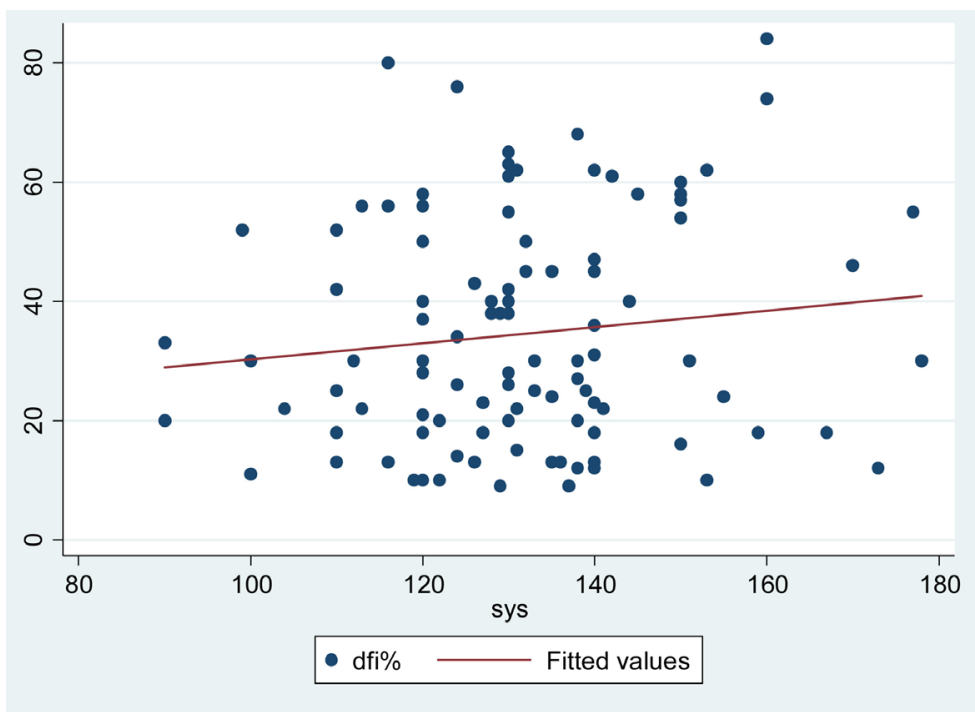

$\mathrm{n}=111 ; \mathrm{y}=16.9+0.14 \mathrm{x} ; \mathrm{R}^{2}=0.0157 ; \mathrm{P}$-value $=0.20$

(This model shows that systolic BP is not reliable to predict the SDFI\%)

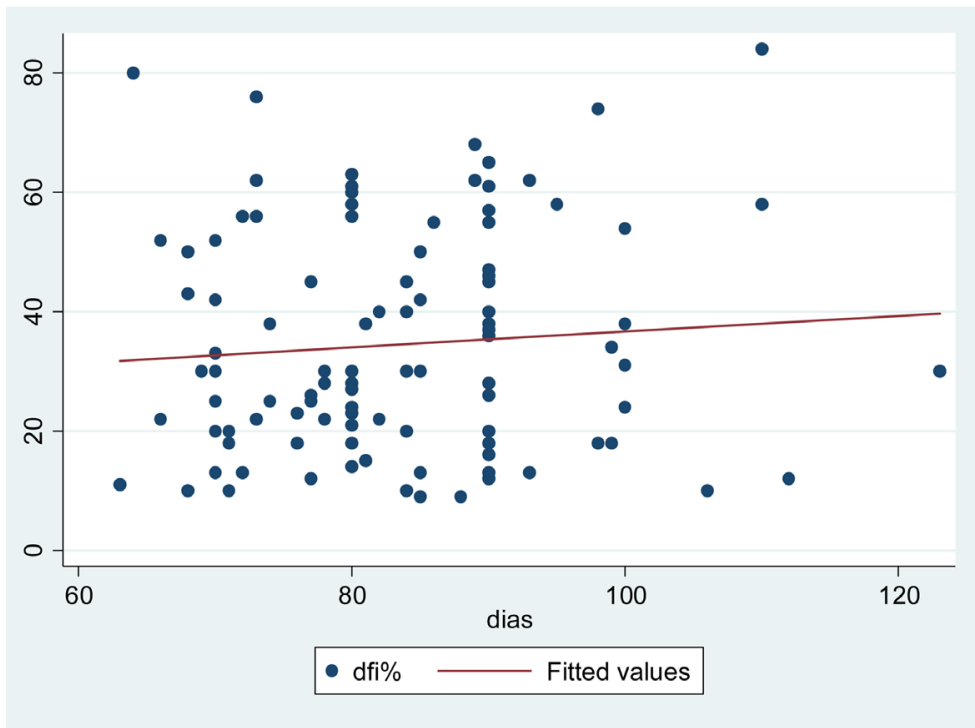

$\mathrm{n}=111 ; \mathrm{y}=23.44+0.13 * x ; \mathrm{R}^{2}=0.0067 ; \mathrm{P}$-value $=0.4590$.

(This model shows that diastolic BP is not reliable to predict the SDFI\%.)

Figure 4. (a) Relationship between SDFI (\%) and Systolic BP (mm.Hg) showing a positive but insignificant correlation using robust standard errors to control for heteroskedasticity. (b) Relationship between SDFI (\%) and Diastolic BP ( $\mathrm{mm} \cdot \mathrm{Hg})$ showing apositive but insignificant correlation using robust standard errors to control for heteroskedasticity.

history of hypertension ( $\mathrm{OR}=0.97,95 \% \mathrm{CI}: 0.43,2.19)$, diabetes $(\mathrm{OR}=0.48$, $95 \%$ CI: $0.05,4.74)$, sexually transmitted illness ( $\mathrm{OR}=0.88,95 \% \mathrm{CI}: 0.33,2.34)$ and mumps (OR $=0.7,95 \%$ CI: $0.13,411)$ to fall in the SDFI $<25 \%$ group (good SDFI) was slim, whereas the odds of falling into the SDFI $\geq 25 \%$ (bad SDFI) group was relatively high.

Correlation matrix for certain variables, relative to DNA fragmentation index, shown in Table 5, indicated that history of infertility, previous groin surgery, 
Table 5. Correlation matrix showing Pearson's $r$ for DNA fragmentation index and other variables.

\begin{tabular}{|c|c|c|c|c|c|c|c|c|c|c|c|}
\hline & $\stackrel{\circ}{\infty}$ & $\sum_{\infty}$ & 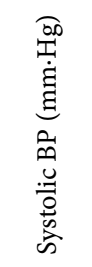 & 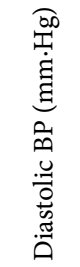 & 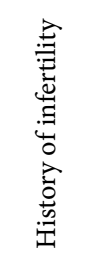 & 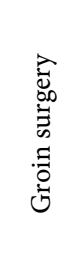 & 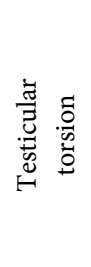 & 害 & 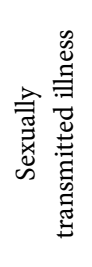 & 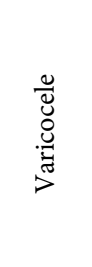 & 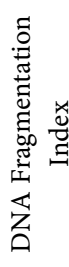 \\
\hline Age & 1.00 & & & & & & & & & & \\
\hline BMI & 0.21 & 1.00 & & & & & & & & & \\
\hline $\begin{array}{c}\text { Systolic BP } \\
(\mathrm{mm} \mathrm{Hg})\end{array}$ & 0.24 & 0.30 & 1.00 & & & & & & & & \\
\hline $\begin{array}{l}\text { Diastolic BP } \\
(\mathrm{mm} \mathrm{Hg})\end{array}$ & 0.19 & 0.25 & 0.69 & 1.00 & & & & & & & \\
\hline History of infertility & -0.47 & -0.08 & -0.03 & -0.01 & 1.00 & & & & & & \\
\hline Groin surgery & 0.20 & -0.03 & 0.04 & -0.03 & -0.16 & 1.00 & & & & & \\
\hline Testicular torsion & 0.11 & 0.10 & 0.11 & 0.04 & -0.06 & 0.39 & 1.00 & & & & \\
\hline Mumps & 0.07 & -0.12 & -0.02 & -0.19 & -0.11 & 0.17 & -0.03 & 1.00 & & & \\
\hline $\begin{array}{c}\text { Sexually } \\
\text { transmitted illness }\end{array}$ & 0.32 & 0.17 & 0.14 & 0.08 & -0.10 & 0.28 & 0.11 & -0.12 & 1.00 & & \\
\hline Varicocele & 0.19 & -0.01 & 0.08 & 0.07 & -0.11 & 0.56 & 0.27 & -0.06 & 0.29 & 1.00 & \\
\hline $\begin{array}{c}\text { DNA } \\
\text { fragmentation Index }\end{array}$ & $0.11^{*}$ & $0.09^{*}$ & $0.06^{*}$ & $0.08^{*}$ & $0.01^{\star}$ & $0.04^{\star}$ & $0.08^{*}$ & $0.04^{\star}$ & $0.04^{\star}$ & $-0.03^{*}$ & 1.00 \\
\hline
\end{tabular}

past infection with mumps and sexually transmitted illness had positive correlations with DNA fragmentation index whereas years trying to conceive, and varicocele had significant but negative correlation with DNA fragmentation index. However, these correlations did not approach any level of significance.

Table 6 shows that, among those who smoke cigarette, SDFI was negatively, but significantly correlated with sperm motility $(\mathrm{r}=-0.30, \mathrm{t}=-2.29, \mathrm{P}$-value $=$ 0.02 , 95\% CI: $-0.55,-0.04)$ while among those who smoke cigarette, SDFI was positively correlated with sperm count $\left(\times 10^{6} / \mathrm{ml}\right)(\mathrm{r}=0.39, \mathrm{t}=4.49, \mathrm{P}$-value $=$ $0.0001,95 \%$ CI: $0.21,0.57)$ but negatively correlated with sperm motility $(\mathrm{r}=$ $-0.59, \mathrm{t}=-3.14, \mathrm{P}$-value $=0.006,95 \% \mathrm{CI}:-0.98,-0.19)$. Alcohol consumption was responsible for a significant $14.42 \%$ variation in the SDFI of the subjects $\left(\mathrm{R}^{2}=\right.$ 0.1442 , Prob $>F=0.0249)$ while cigarette smoking explained a significant $33.61 \%$ variation in the SDFI of subjects $\left(\mathrm{R}^{2}=0.3361\right.$, Prob $\left.>F=0.0031\right)$.

SDFI had a positive and statistically significant correlation only with age among non-consumers of alcohol $(\mathrm{r}=0.73, \mathrm{t}=2.70, \mathrm{P}$-value $=0.01,95 \% \mathrm{CI}$ : $0.18,1.28)$ but a statistically significant negative correlation only with sperm motility among those who consume alcohol $(\mathrm{r}=-0.42, \mathrm{t}=-2.88$, P-value $=$ 0.006, 95\% CI: $-0.71,-0.13$ ) (Table 7). 
Table 6. Robust linear regression analysis of SDFI as dependent variable against independent variables of age, BMI, TTC, Count and Motility among smokers and non-smokers.

\begin{tabular}{|c|c|c|c|c|c|}
\hline \multicolumn{6}{|c|}{ Do not smoke cigarette $(\mathrm{n}=88)$} \\
\hline$F(5,82)=1.60$ & Prob $>F=0.17$ & $\mathrm{R}^{2}=0.1010$ & & Root $\mathrm{N}$ & $=18.75$ \\
\hline SDFI & Coef. & SE & $\mathrm{t}$ & $P>[t]$ & $\begin{array}{l}\text { 95\% Confidence } \\
\text { Interval }\end{array}$ \\
\hline Age & 0.26 & 0.25 & 1.01 & 0.32 & $-0.25,0.76$ \\
\hline BMI & 0.33 & 0.51 & 0.64 & 0.52 & $-0.69,1.35$ \\
\hline $\begin{array}{l}\text { Years trying } \\
\text { to conceive }\end{array}$ & -0.47 & 0.36 & -1.30 & 0.20 & $-1.18,0.25$ \\
\hline Count & 0.02 & 0.09 & 0.19 & 0.85 & $-0.17,0.40$ \\
\hline Motility & -0.30 & 0.13 & -2.29 & 0.02 & $-0.55,-0.04$ \\
\hline _cons & 29.87 & 16.25 & 1.84 & 0.07 & $-2.46,62.19$ \\
\hline \multicolumn{6}{|c|}{ Smoke cigarette $(n=23)$} \\
\hline$F(5,17)=5.62$ & Prob $>F=0.0031$ & $\mathrm{R}^{2}=0.3361$ & & Root $N$ & $=15.60$ \\
\hline SDFI & Coef. & SE & $\mathrm{t}$ & $P>[t]$ & $\begin{array}{l}\text { 95\% Confidence } \\
\text { Interval }\end{array}$ \\
\hline Age & 0.28 & 0.28 & 0.97 & 0.34 & $-0.32,0.88$ \\
\hline BMI & -0.26 & 0.64 & -0.40 & 0.69 & $-1.62,1.10$ \\
\hline $\begin{array}{l}\text { Years trying } \\
\text { to conceive }\end{array}$ & -1.28 & 0.65 & -1.96 & 0.07 & $-2.660,0.10$ \\
\hline Count & 0.39 & 0.09 & 4.49 & 0.0001 & $0.21,0.57$ \\
\hline Motility & -0.59 & 0.19 & -3.14 & 0.006 & $-0.98,-0.19$ \\
\hline _cons & 53.58 & 24.45 & 2.19 & 0.043 & $1.99,105.17$ \\
\hline
\end{tabular}

\section{Discussion}

In almost all cases, the laboratory diagnosis of male infertility in sub-Saharan Africa is mainly based on the conventional seminal fluid analysis [24] [25], which is incapable of detecting the delicate aberrations in the male genome characterized by damaged sperm DNA [25] [26] [27]. Interestingly, Altura et al., have linked sperm DNA fragmentation to Magnesium deficiency in an animal model [28] which may be projected into human model because most diets, especially in Africa, may be magnesium-deficient. Three major conclusions can be drawn from this study which, to our knowledge, is the first ever-reported study on the human sperm DNA fragmentation among Black Africans. First, and most importantly, the prevalence of SDFI $\geq 25 \%$ was generally higher than that of SDFI $<25 \%$ among the study subjects. The mean sperm DNA fragmentation of $34.5 \%$ reported in this study is higher than the $27.6 \%$ reported by Majzoub et al., in a Qatari study [29]. Fragmentation of sperm DNA is multifactorial, ranging from social habits, lifestyle and systemic illness such as diabetes among others. This study has identified the possibility of DNA fragmentation among men with high blood pressure and among those with history of diabetes mellitus, two major systemic diseases that are ravaging Black Africans in recent history. This study speculates that DNA fragmentation seen in those with high systolic and 
Table 7. Robust Linear Regression analysis of SDFI as dependent variable against independent variables of age, BMI, TTC, Count and Motility among alcohol consumers and non-consumers.

\begin{tabular}{|c|c|c|c|c|c|}
\hline \multicolumn{6}{|c|}{ Do not consume alcohol $(\mathrm{n}=42)$} \\
\hline$F(5,36)=2.90$ & Prob $>F=0.0269$ & $\mathrm{R}^{2}=0.1936$ & & Root $\mathrm{I}$ & $=16.38$ \\
\hline SDFI & Coef. & SE & $\mathrm{t}$ & $\mathrm{P}>[\mathrm{t}]$ & $\begin{array}{c}\text { 95\% Confidence } \\
\text { Interval }\end{array}$ \\
\hline Age & 0.73 & 0.27 & 2.70 & 0.01 & $0.18,1.28$ \\
\hline BMI & -0.48 & 0.74 & -0.64 & 0.52 & $-1.98,1.02$ \\
\hline $\begin{array}{l}\text { Years trying } \\
\text { to conceive }\end{array}$ & -0.39 & 0.46 & -0.85 & 0.40 & $-1.31,0.54$ \\
\hline Count & -0.06 & 0.13 & -0.49 & 0.63 & $-0.32,0.19$ \\
\hline Motility & -0.20 & 0.16 & -1.26 & 0.22 & $-0.53,0.12$ \\
\hline _cons & 25.20 & 23.43 & 1.08 & 0.29 & $-22.33,72.72$ \\
\hline \multicolumn{6}{|c|}{ Consume alcohol $(n=69)$} \\
\hline$F(5,63)=2.78$ & Prob $>F=0.0249$ & $\mathrm{R}^{2}=0.1442$ & \multicolumn{3}{|c|}{ Root MSE $=18.48$} \\
\hline SDFI & Coef. & SE & $\mathrm{t}$ & $\mathrm{P}>[\mathrm{t}]$ & $\begin{array}{c}\text { 95\% Confidence } \\
\text { Interval }\end{array}$ \\
\hline Age & 0.00 & 0.29 & 0.00 & 1.00 & $-0.58,0.58$ \\
\hline BMI & 0.22 & 0.56 & 0.39 & 0.70 & $-0.90,1.34$ \\
\hline $\begin{array}{l}\text { Years trying } \\
\text { to conceive }\end{array}$ & -0.68 & 0.38 & -1.81 & 0.08 & $-1.43,0.07$ \\
\hline Count & 0.10 & 0.10 & 1.05 & 0.30 & $-0.09,0.30$ \\
\hline Motility & -0.42 & 0.15 & -2.88 & 0.006 & $-0.71,-0.13$ \\
\hline _cons & 49.65 & 16.98 & 2.92 & 0.005 & $15.73,83.58$ \\
\hline
\end{tabular}

diastolic blood pressure, may have been modulated via the high-sensitivity C-reactive protein (hsCRP), an inflammatory bio-marker detected in acute coronary syndrome and stable coronary artery disease (CAD) [30] [31] [32]. Kotani and Sakane's study also concluded that patients with metabolic syndrome-which includes high blood pressure-may have a closer linkage with inflammation and oxidative stress than those without metabolic syndrome [33]. Agawal and Wang reported that levels of Oxidation-Reduction potential (ORP), probably indicator of oxidative stress, were significantly elevated in semen samples with abnormal sperm parameters [34]. Oxidation-Reduction Potential stress is associated with DNA fragmentation [34] and oxidative stress, engendered by high ORP and Reactive oxygen species, during episodes of high blood pressure, may be responsible for nicking the sperm DNA. The mean SDFI \% of subjects with high systolic $(\geq 140 \mathrm{~mm} \cdot \mathrm{Hg})$ and diastolic $(\geq 90 \mathrm{~mm} \cdot \mathrm{Hg})$ blood pressures were higher than those of subjects with normal (systolic $<140 \mathrm{~mm} \cdot \mathrm{Hg}$ ) and diastolic $(<90$ $\mathrm{mm} \cdot \mathrm{Hg}$ ) BP, though the differences were statistically insignificant. It therefore seems that high blood pressure level may be deleterious to sperm DNA integrity, possibly through several convoluted pathways. Further studies are needed to clarify this point. 
Sperm DNA fragmentation index (\%) was significantly higher in obese men than in overweight or normal men. In fact, the proportion of normal men with SDFI $<25 \%$ (good SDFI) was higher than of men with SDFI $\geq 25 \%$ (bad SDFI); the proportion of overweight men with SDFI $<25 \%$ (good SDFI) was similar to that of men with SDFI $\geq 25 \%$ (bad SDFI); and the proportion of obese men with SDFI $\geq 25 \%$ (bad SDFI) was higher than of men with SDFI $<25 \%$ (good SDFI). Fariello et al., also reported a higher percentage of sperm with high DNA fragmentation ( $(\mathrm{P}=0.004)$ among obese subjects [35] an observation that was validated by Dupont et al., in their works [36]. The disturbance of spermatogenesis might be one of the mechanisms by which excess fat tissue has a negative impact on male fertility [37]. Production of abnormal reproductive hormone levels, increased release of adipose-derived hormones and adipokines associated with obesity, as well as some physical complications such as sleep apnea and high scrotal temperatures may be responsible for elucidating the consequence of obesity on male infertility [15].

Social habits have also been linked to sperm DNA fragmentation. For example, the mean SDFI\% among subject who reported to consume alcohol (37.1 \pm 19.2) was significantly higher than that of subjects who reported non-consumption of alcohol $(30.2 \pm 17.1)$, a figure comparable to the median SDFI of $42.50 \%$ reported by Wdowiak et al. [17], the $49.6 \% \pm 23.3 \%$ documented by Komiya et al. [38] and similar reports in other human [18] [39] and animal studies [40] [41]. That subjects who claimed not to smoke would record higher SDFI\% than those who claimed to smoke was surprising. However, it is possible that though some people may not smoke, they could be exposed to the cigarette fumes of those who smoke.

Alcohol consumption supposedly damages sperm DNA integrity possibly as a result of the oxidative stress generated by the ethanol as alcohol-induced oxidative stress might be deleterious not only to the liver, but also to other extrahepatic tissues and organs of the body, including the testes [42] [43] [44] [45]. Chronic consumption of ethanol might be incriminated in endocrine and reproductive failure via testicular lipid peroxidation, reductions in the content of polyenoic fatty acids and glutathione (GSH) of the testes, membrane injury and dysfunctional gonads [46] [47] [48] [49]. From another perspective, oxidative stress may be induced via increased conversion of xanthine dehydrogenase into xanthine oxidase, and the activation of peroxisomal acyl CoA-oxidase linked to the consumption of ethanol may be a contributing factor to oxidative stress [17].

\section{Conclusion}

Data from this study indicate that the infertile men had significantly higher sperm DNA fragmentation, especially among obese men and those who consume alcohol. Moreover, it appears that sperm nuclear DNA fragmentation may increase with age and with systolic and diastolic blood pressures. There are several studies that have proposed various mechanisms and factors that probably 
cause sperm DNA fragmentation. Most of these studies relate SDF to male infertility [18] [50] [51] [52]. Studies on sperm DNA fragmentation among indigenous Black African population should be vigorously undertaken to benefit men who are not able to father a child. Further, more studies should be carried out on the clinical efficacy and advantage of SDFI in male infertility and in Assisted Reproduction Technology.

\section{Study Limitations}

This study has certain limitations that need to be discussed. First, the sampling methodology might be biased against other groups of people. This study sampled only men who presented with infertility. The sperm DNA fragmentation in apparently fertile men was not examined. Also, this was a fertility-based study and the sample size was small, therefore, conclusions on SDF in the general public cannot be drawn from this data alone. Next, responses on social habits such as alcohol consumption, cigarette smoking and use of herbal teas were just Yes or No and there was no assessment of quantity, concentration or duration of consumption of these substances. We also did not measure blood sugar level concentration to determine whether subjects were diabetic or not. Further, there was no patient with BMI $<185 \mathrm{~kg} / \mathrm{m}^{2}$ making it impossible to determine SDF among this group of people. Also, SDF was not reported in this study, from the perspective of occupation or exposure to environmental toxins. Although many variables such as age, alcohol cigarette smoking, and high body mass index can impact sperm DNA integrity, we did not perform unadjusted and adjusted odds ratio analysis to control for any or all of these factors.

\section{Conflicts of Interest}

None declared by any and all of the authors.

\section{References}

[1] Gurunath, S., Pandian, Z., Anderson, R.A. and Bhattacharya, S. (2001) Defining Infertility-As Systematic Review of Prevalence Studies. Human Reproduction Update, 17, 575-588. https://doi.org/10.1093/humupd/dmr015

[2] World Health Organization (1999) Laboratory Manual for the Examination of Human Semen and Sperm-Cervical Mucucs Interaction. 4th Edition, Cambridge University Press, Cambridge.

[3] World Health Organization (2000) WHO Manual for the Standardized Investigation and Diagnosis of the Infertile Couple. Cambridge University Press, Cambridge.

[4] Shange, N. Male Infertility in Africa: Breaking the Stigma. https://www.sowetanlive.co.za/good-life/health/2018-07-16-male-infertility-in-afric a-breaking-the-stigma/

[5] Kersian, M. https://medium.com/@Episona/what-is-sperm-dna-fragmentation-5c7245591570

[6] Jequier, A.M. (2004) Clinical Andrology-Still a Major Problem in the Treatment of Infertility. Human Reproduction, 19, 1245-1249. 
https://doi.org/10.1093/humrep/deh269

[7] Cho, C.L. and Agarwal, A. (2017) Role of Sperm DNA Fragmentation in Male Factor Infertility: A Systematic Review. Arab Journal of Urology, 16, 21-34.

[8] Bradley, C.K., McArthur, S.J., Gee, A.J., Weiss, K.A., Schmidt, U. and Toogood, L. (2016) Intervention Improves Assisted Conception Intracytoplasmic Sperm Injection Outcomes for Patients with High Levels of Sperm DNA Fragmentation: A Retrospective Analysis. Andrology, 4, 903-910. https://doi.org/10.1111/andr.12215

[9] Gavriliouk, D. and Aitken, R.J. (2015) Damage to Sperm DNA Mediated by Reactive Oxygen Species: Its Impact on Human Reproduction and the Health Trajectory of Off spring. Advances in Experimental Medicine and Biology, 868, 23-47. https://doi.org/10.1007/978-3-319-18881-2_2

[10] Aitken, R.J., Smith, T.B., Jobling, M.S., Baker, M.A. and De Iuliis, G.N. (2014) Oxidative Stress and Male Reproductive Health. Asian Journal of Andrology, 16, 31-38. https://doi.org/10.4103/1008-682X.122203

[11] Wright, C., Milne, S. and Leeson, H. (2014) Sperm DNA Damage Caused by Oxidative Stress: Modifiable Clinical, Lifestyle and Nutritional Factors in Male Infertility. Reproductive BioMedicine Online, 28, 684-703. https://doi.org/10.1016/j.rbmo.2014.02.004

[12] Gunes, S., Al-Sadaan, M. and Agarwal, A. (2015) Spermatogenesis, DNA Damage and DNA Repair Mechanisms in Male Infertility. Reproductive BioMedicine Online, 31, 309-319. https://doi.org/10.1016/j.rbmo.2015.06.010

[13] Humm, K.C. and Sakkas, D. (2013) Role of Increased Male Age in IVF and Egg Donation: Is Sperm DNA Fragmentation Responsible? Fertility and Sterility, 99, 30-36. https://doi.org/10.1016/j.fertnstert.2012.11.024

[14] Boscoa, L., Notari, T., Ruvolo, G., Roccheri, M.C., Martino, C., Chiappetta, R., Carone, D., Bosco, G., Carrillo, L., Raimondo, S., Guglielmino, A. and Montano, L. (2018) Sperm DNA Fragmentation: An Early and Reliable Marker of Air Pollution. Environmental Toxicology and Pharmacology, 58, 243-249.

https://doi.org/10.1016/j.etap.2018.02.001

[15] Cabler, S., Agarwal, A., Flint, M. and Du Plessis, S. (2010) Obesity: Modern Man's Fertility Nemesis. Asian Journal of Andrology, 12, 480-489. https://doi.org/10.1038/aja.2010.38

[16] Gilbert, B.R. https://brucegilbertmd.com/3262014-looks-can-deceiving-sperm-dna-fragmentatio n-male-fertility/

[17] Wdowiak, A., Wdowiak, A., Bakalczuk, S. and Bakalczuk, G. (2016) Relationship between Alcohol Consumption and Sperm Nuclear DNA Fragmentation and Pregnancy. Advances in Andrology Online, 3, 14-21.

[18] Anifandis, G., Bounartzi, T., Messini, C.I., Dafopoulos, K., Sotiriou, S. and Messinis, I.E. (2014) The Impact of Cigarette Smoking and Alcohol Consumption on Sperm Parameters and Sperm DNA Fragmentation (SDF) Measured by Halosperm. Archives of Gynecology and Obstetrics, 290, 777. https://doi.org/10.1007/s00404-014-3281-x

[19] Sharif, K. (2013) Sperm DNA Fragmentation Testing: To Do or Not to Do? Middle East Fertility Society Journal, 18, 78-83. https://doi.org/10.1016/j.mefs.2013.02.001

[20] Lewis Sheena, E.M., John Aitken, R., Conner, S.J., De Iuliis, G., Evenson, D.P., Henkel, R., Giwercman, A. and Gharagozloo, P. (2013) The Impact of Sperm DNA Damage in Assisted Conception and Beyond: Recent Advances in Diagnosis and 
Treatment. Reproductive BioMedicine Online, 27, 325-337.

[21] Lewis, S.E.M. (2013) The Place of Sperm DNA Fragmentation Testing in Current Day Fertility Management. Middle East Fertility Society Journal, 18, 78-83. https://doi.org/10.1016/j.mefs.2013.01.010

[22] Fernández, J.L., Muriel, L., Rivero, M.T., Goyanes, V., Vazquez, R. and Alvarez, J.G. (2003) The Sperm Chromatin Dispersion Test: A Simple Method for the Determination of Sperm DNA Fragmentation. Journal of Andrology, 24, 59-66.

[23] TDL Andrology. Information about Sperm DNA Fragmentation for Clinicians. https://tdlpathology.com/services-divisions/tdl-andrology/sperm-dnafragmentation

[24] Simon, L., Brunborg, G., Stevenson, M., Lutton, D., McManus, J., et al. (2010) Clinical Significance of Sperm DNA Damage in Assisted Reproduction Outcome. Human Reproduction, 25, 1594-1608. https://doi.org/10.1093/humrep/deq103

[25] Esteves, S.C. and Agarwal, A. (2011) Novel Concepts in Male Infertility. International Brazilian Journal of Urology, 37, 5-15. https://doi.org/10.1590/S1677-55382011000100002

[26] Piasecka, M., Gaczarzewicz, D. and Laszczyriska, M. (2006) Evaluation of Sperm Genomic Integrity of Normozoospermic Men: A Prospective Study. Folia Histochemica et Cytobiologica, 44, 117-122.

[27] Micinski, P., Pawlicki, K., Wielgus, E., Bochenek, M., Gogol, P., et al. (2011) Total Reactive Antioxidant Potential and DNA Fragmentation Index as Fertility Sperm Parameters. Reproductive Biology, 11, 135-144. https://doi.org/10.1016/S1642-431X(12)60050-3

[28] Altura, B.M., Shah, N.C., Shah, G.J., Pérez-Abela, J.L. and Altura, B.T. (2016) Magnesium Deficiency Results in Oxidation and Fragmentation of DNA, Down Regulation of Telomerase Activity, and Ceramide Release in Cardiovascular Tissues and Cells: Potential Relationship to Atherogenesis, Cardiovascular Diseases and Aging. International Journal of Diabetology \& Vascular Disease Research, 4, 1-5.

[29] Majzoub, A., Arafa, M., Mahdi, M., Agarwal, A., Al Said, S., Al-Emadi, I., El Ansari, W., Alattar, A., Al Rumaihi, K. and Elbardisi, H. (2018) Oxidation-Reduction Potential and Sperm DNA Fragmentation, and Their Associations with Sperm Morphological Anomalies amongst Fertile and Infertile Men. Arab Journal of Urology, 16, 87-95. https://doi.org/10.1016/j.aju.2017.11.014

[30] Liuzzo, G., Biasucci, L.M., Gallimore, J.R., Grillo, R.L., Rebuzzi, A.G., Pepys, M.B. and Maseri, A. (1994) The Prognostic Value of C-Reactive Protein and Serum Amyloid a Protein in Severe Unstable Angina. The New England Journal of Medicine, 331, 417-424. https://doi.org/10.1056/NEJM199408183310701

[31] Ridker, P.M. (2001) High-Sensitivity C-Reactive Protein: Potential Adjunct for Global Risk Assessment in the Primary Prevention of Cardiovascular Disease. Circulation, 103, 1813-1818. https://doi.org/10.1161/01.CIR.103.13.1813

[32] Ridker, P.M., Cushman, M., Stampfer, M.J., Tracy, R.P. and Hennekens, C.H. (1997) Inflammation, Aspirin, and the Risk of Cardiovascular Disease in Apparently healthy Men. The New England Journal of Medicine, 336, 973-979. https://doi.org/10.1056/NEJM199704033361401

[33] Kotani, K. and Sakane, N. (2012) C-Reactive Protein and Reactive Oxygen Metabolites in Subjects with Metabolic Syndrome. The Journal of International Medical Research, 40, 1074-1081. https://doi.org/10.1177/147323001204000326

[34] Agarwal, A. and Wang, S.M. (2017) Clinical Relevance of Oxidation-Reduction Potential in the Evaluation of Male Infertility. Urology, 104, 84-89. 
https://doi.org/10.1016/j.urology.2017.02.016

[35] Fariello, R.M., Pariz, J.R., Spaine, D.M., Cedenho, A.P., Bertolla, R.P. and Fraietta, R. (2012) Association between Obesity and Alteration of Sperm DNA Integrity and Mitochondrial Activity. BJU International, 110, 863-867. https://doi.org/10.1111/j.1464-410X.2011.10813.x

[36] Dupont, C., Faure, C., Sermondade, N., Boubaya, M., Eustache, F., Clement, P., Pascal Briot, P., et al. (2013) Obesity Leads to Higher Risk of Sperm DNA Damage in Infertile Patients. Asian Journal of Andrology, 15, 622-625. https://doi.org/10.1038/aja.2013.65

[37] Vitiazeva, I.I., Altashina, M.V., Mun, T.V. and Troshina, E.A. (2015) Influence of Obesity on Sperm DNA Fragmentation Index and Outcomes of IVF Programs. Problems of Endocrinology, 61, 48-55. https://doi.org/10.14341/probl201561548-55

[38] Komiya, A., Kato, T., Kawauchi, Y., Watanabe, A. and Fuse, H. (2014) Clinical Factors Associated with Sperm DNA Fragmentation in Male Patients with Infertility. The Scientific World Journal, 2014, Article ID: 868303.

[39] Vellani, E., Colasante, A., Mamazza, L., Minasi, M.G., Greco, E. and Bevilacqua, A. (2013) Association of State and Trait Anxiety to Semen Quality of in Vitro Fertilization Patients: A Controlled Study. Fertility and Sterility, 99, 1565-1572. https://doi.org/10.1016/j.fertnstert.2013.01.098

[40] Pourentezari, M., Talebi, A.R., Mangoli, E., Anvari, M. and Rahimipour, M. (2016) Additional Deleterious Effects of Alcohol Consumption on Sperm Parameters and DNA Integrity in Diabetic Mice. Andrologia, 48, 564-569.

https://doi.org/10.1111/and.12481

[41] Talebi, A.R., Sarcheshmeh, A.A., Khalili, M.A. and Tabibnejad, N. (2011) Effects of Ethanol Consumption on Chromatin Condensation and DNA Integrity of Epididymal Spermatozoa in Rats. Alcohol, 45, 403-409.

https://doi.org/10.1016/j.alcohol.2010.10.005

[42] Galicia-Moreno, M. and Gutiérrez-Reyes, G. (2014) The Role of Oxidative Stress in the Development of Alcoholic Liver Disease. Revista de Gastroenterología de México, 79, 135-144. https://doi.org/10.1016/j.rgmx.2014.03.001

[43] Ozbek, E. (2012) Induction of Oxidative Stress in Kidney. International Journal of Nephrology, 2012, Article ID: 465897. https://doi.org/10.1155/2012/465897

[44] Rosenblum, E.R., Gavaler, J.S. and Van Thiel, D.H. (1989) Lipid Peroxidation: A Mechanism for Alcohol Induced Testicular Injury. Free Radical Biology \& Medicine, 7, 569-577. https://doi.org/10.1016/0891-5849(89)90034-8

[45] Zakhari, S. (2006) Overview: How Is Alcohol Metabolized by the Body? Alcohol Research \& Health, 29, 245-254.

[46] Dosumu, O.O., Osinubi, A.A. and Duru, F.I.O. (2014) Alcohol Induced Testicular Damage: Can Abstinence Equal Recovery? Middle East Fertility Society Journal, 19, 221-228. https://doi.org/10.1016/j.mefs.2014.01.003

[47] Himabindua, B., Madhua, P. and Sreenivasula-Reddy, P. (2015) Diabetes and Alcohol: Double Jeopardy with Regard to Oxidative Toxicity and Sexual Dysfunction in Adult Male Wistar Rats. Reproductive Toxicology, 51, 57-63. https://doi.org/10.1016/j.reprotox.2014.12.010

[48] La Vignera, S., Condorelli, R.A., Balercia, G., Vicari, E. and Calogero, A.E. (2013) Does Alcohol Have Any Effect on Male Reproductive Function? A Review of Literature. Asian Journal of Andrology, 15, 221-225.

https://doi.org/10.1038/aja.2012.118 
[49] Nordmann, R. (1994) Alcohol and Antioxidant Systems. Alcohol, 29, 513-522.

[50] Frączek, M. and Kurpisz, M. (2015) Mechanisms of the Harmful Effects of Bacterial Semen Infection on Ejaculated Human Spermatozoa: Potential Inflammatory Markers in Semen. Folia Histochemica et Cytobiologica, 53, 201-217. https://doi.org/10.5603/fhc.a2015.0019

[51] Morris, I.D., Ilott, S., Dixon, L. and Brison, D.R. (2002) The Spectrum of DNA Damage in Human Sperm Assessed by Single Cell Gel Electrophoresis (Comet Assay) and Its Relationship to Fertilization and Embryo Development. Human Reproduction, 17, 990-908. https://doi.org/10.1093/humrep/17.4.990

[52] Singh, N.P., Muller, C.H. and Berger, R.E. (2003) Effects of Age on DNA Double Strand Breaks and Apoptosis in Human Sperm. Fertility and Sterility, 80, 1420-1430. https://doi.org/10.1016/j.fertnstert.2003.04.002

\section{Abbreviations}

$\mathrm{WHO}=$ World health organization; DNA = Deoxyribonucleic acid; SFA $=$ Seminal Fluid Analysis; SDF $=$ Sperm DNA Fragmentation; ART $=$ Assisted Reproduction Technology; BMI = Body Mass Index; SCD = Sperm chromatin dispersion; DAPI =; PBS = Phosphate-buffered saline; SDFI = DNA fragmentation index. NFC = Nordica Fertility Center; ANOVA = Analysis of Variance; OR = Odds Ratio; $\mathrm{CI}=$ Confidence Interval; ORP = Oxidation-Reduction Potential; $\mathrm{mm} \cdot \mathrm{Hg}=$ millimeter of Mercury. 